\title{
Webinar to Promote Mental Wellness Among Healthcare Staff During the COVID-19 Pandemic
}

\author{
J Chung, WS Yeung
}

To the Editor:

Mental health risks increase during the pandemic, ${ }^{1}$ owing to unpredictability and uncertainty, social distancing, loss of income, increase in alcohol use, and online gambling. ${ }^{2}$ In a survey of 1257 healthcare workers in China, 50.4\% reported symptoms of depression, $44.6 \%$ reported symptoms of anxiety, $34.0 \%$ reported symptoms of insomnia, and $71.5 \%$ reported symptoms of distress. ${ }^{3}$

Healthcare professionals may be reluctant to seek care for mental health conditions. $40 \%$ of physicians reported that they would be reluctant to seek formal medical care for treatment of a mental health condition because of concerns about repercussions to their medical licensure. ${ }^{4}$ Primary prevention is important to reduce mental health risks in healthcare professionals.

In February 2020, the Hong Kong East Cluster initiated a comprehensive support programme named Support of You to provide mental health information and online selfassessment for staff, which is especially important during the coronavirus disease 2019 (COVID-19) pandemic.

Webinars are an effective way to disseminate knowledge, with participants rating them highly in terms of ability to gain knowledge and intention to share the knowledge gained. ${ }^{5}$ Webinars also have higher participation rates than physical workshops do because of the convenience of online learning. Among 326 medical and nursing staff of the Department of Psychiatry in Hong Kong East Cluster, the average participation rate during the COVID-19 pandemic was $13 \%$ for lunch time training workshops and $16 \%$ for lunch time training webinars.

In August 2020, the Support of You programme delivered a webinar to promote mental wellness and build resilience among healthcare staff. The 2-hour webinar was divided into five sessions: mental health information, parenting during the pandemic, stay-at-home activities, mental health self-care techniques, and play skills for parents with small children. Of 112 staff (71 were nurses) registered for the webinar, 105 (93.8\%) logged in to the webinar. The mean time stayed in the webinar was 112 minutes, and 71 (67.6\%) participants stayed for $>1.5$ hours. 35 (33.3\%) participants completed the online post-webinar evaluation, in which items were rated using a 5-point Likert scale from 1 (strongly agree) to 5 (strongly disagree). For all evaluation questions, $>75 \%$ of respondents strongly agreed or agreed that the webinar was useful and favourably received (Table).

The webinar was recorded and shared with all healthcare staff at Hong Kong East Cluster hospitals through

Table. Results of a survey conducted after a webinar to promote mental wellness and build resilience among healthcare $\operatorname{staff}(n=35)$

\begin{tabular}{|lc|}
\hline & $\begin{array}{c}\text { No. (\%) of } \\
\text { respondents } \\
\text { strongly agreed or } \\
\text { agreed }\end{array}$ \\
\hline The webinar helped improve my understanding about mental health problems during COVID-19 & $30(85.7)$ \\
\hline The webinar helped improve my own mental health during COVID-19 & $27(77.1)$ \\
\hline The webinar gave me useful information to improve family relationship & $29(82.9)$ \\
\hline The webinar improved my knowledge about community mental health resources & $27(77.1)$ \\
\hline The webinar improved my understanding about how to build resilience during COVID-19 & $29(82.9)$ \\
\hline The webinar increased my motivation to nourish my own mental health during COVID-19 & $29(82.9)$ \\
\hline The video quality of the webinar was good & $30(85.7)$ \\
\hline The sound quality of the webinar was good & $30(85.7)$ \\
\hline Compared to physical workshop, webinar did not compromise learning & $28(80.0)$ \\
\hline During the COVID-19 pandemic, webinar is preferred instead of physical workshop/staff forum & $32(91.4)$ \\
\hline I wish Hong Kong East Cluster can organise more webinars & $30(85.7)$ \\
\hline
\end{tabular}


emails and hospital websites, so that even those who did not join the webinar can benefit by watching the video. There are plans to organise more webinars to target the specific mental health needs. To increase the participation rate, a pre-webinar survey about staff interests and needs would be helpful. More promotion is also needed to encourage staff to embrace this new form of learning.

Most participants were nurses. Nurses experienced more severe mental health symptoms than other healthcare workers during the COVID-19 pandemic. ${ }^{3}$ Their needs should be considered in the planning of webinars. Topics should cover not only mental health issues but also the psychosocial needs of healthcare professionals. Support from information technology staff is important to ensure the smooth running of webinars. Other hospitals in Hong Kong should consider using webinars to deliver mental health information to healthcare staff to improve their resilience during the COVID-19 pandemic.

\section{Declaration}

The authors have no conflict of interests to disclose.

Joseph Chung, Department of Psychiatry, Pamela Youde Nethersole Eastern Hospital, Hong Kong
Wai-Song Yeung, Department of Psychiatry, Pamela Youde Nethersole Eastern Hospital, Hong Kong

Address for correspondence: Dr Joseph Chung, Department of Psychiatry, Pamela Youde Nethersole Eastern Hospital, 3 Lok Man Road, Chai Wan, Hong Kong.

Email: chungpy2@ha.org.hk

Submitted: 6 August 2020; Accepted: 18 November 2020

\section{References}

1. Shi L, Lu ZA, Que JY, Huang XL, Liu L, Ran MS, et al. Prevalence of and risk factors associated with mental health symptoms among the general population in China during the coronavirus disease 2019 pandemic. JAMA Netw Open 2020;3:e2014053. Crossref

2. Moreno C, Wykes T, Galderisi S, Nordentoft M, Crossley N, Jones N, et al. How mental health care should change as a consequence of the COVID-19 pandemic. Lancet Psychiatry 2020;7:813-24. Crossref

3. Lai J, Ma S, Wang Y, Cai Z, Hu J, Wei N, et al. Factors associated with mental health outcomes among health care workers exposed to coronavirus disease 2019. JAMA Netw Open 2020;3:e203976. Crossref

4. Dyrbye LN, West CP, Sinsky CA, Goeders LE, Satele DV, Shanafelt TD. Medical licensure questions and physician reluctance to seek care for mental health conditions. Mayo Clin Proc 2017;92:1486-93. Crossref

5. Hoke AM, Francis EB, Hivner EA, Simpson AJL, Hogentogler RE, Kraschnewski JL. Investigating the effectiveness of webinars in the adoption of proven school wellness strategies. Health Educ J 2018;77:249-57. Crossref 